\title{
Tetraband Small-Size Printed Strip MIMO Antenna for Mobile Handset Application
}

\author{
Qinghao Zeng, ${ }^{1}$ Yuan Yao, ${ }^{1}$ Shaohua Liu, ${ }^{1}$ Junsheng Yu, ${ }^{1}$ Peng Xie, ${ }^{1}$ and Xiaodong Chen ${ }^{2}$ \\ ${ }^{1}$ School of Electronic Engineering, Beijing University of Posts and Telecommunications, Beijing 100876, China \\ ${ }^{2}$ School of Electronic Engineering and Computer Science, Queen Mary University of London, London E1 4NS, UK
}

Correspondence should be addressed to Qinghao Zeng, hankszeng@163.com

Received 31 August 2011; Accepted 10 October 2011

Academic Editor: Li Yang

Copyright (C) 2012 Qinghao Zeng et al. This is an open access article distributed under the Creative Commons Attribution License, which permits unrestricted use, distribution, and reproduction in any medium, provided the original work is properly cited.

\begin{abstract}
A compact printed multiple-input multiple-output (MIMO) antenna for tetraband (GSM900/1800/1900/UMTS) mobile handset application is presented. The proposed MIMO antenna, which consists of two coupled-fed loop antennas with symmetrical configuration, was printed on a $120 * 60 * 0.8 \mathrm{~mm}^{3} \mathrm{Fr}-4$ substrate of relative permittivity of 4.4 , loss tangent 0.02 . Each element antenna requires only a small area of $22.5 * 25 \mathrm{~mm}^{2}$ on the circuit board. The edge-to-edge spacing between the two elements is only $0.03 \lambda_{0}$ of $920 \mathrm{MHz}$. A slot and a dual-inverted-L-shaped ground branch were added in the ground plane to decrease the mature coupling between the antenna elements. The measured isolation of the proposed antenna is better than $15 \mathrm{~dB}$ among the four operating frequency bands. The simulated 3D radiation patterns at $900 \mathrm{MHz}$ and $1900 \mathrm{MHz}$ of both antenna elements show that two loop antennas in general cover complementary space regions with good diversity performance. Detailed antenna impedance matching performance comparisons were done to evaluate the benefit of using different decoupling technology. The envelop correlation coefficient is calculated to represent the diversity performance of the MIMO antenna.
\end{abstract}

\section{Introduction}

Multiple-input and multiple-output (MIMO) technology which seemed as a key technology for long-term evolution (LTE) has attracted significant attention [1-3]. Theoretical and experimental investigations have revealed substantial improvements in channel capacity and reliability in rich scattering environments when multiple transmitter and receiver antennas are deployed $[4,5]$. There are more limitations for engineers to design a qualified MIMO antenna for handset applications than a MIMO antenna for base station applications such as the extremely small size and the mutual coupling between the element antennas $[6,7]$. The correlation coefficient is directly related to the mutual coupling between the element antennas. The higher the isolation was, the higher the data transmission speed could be obtained [8].

Many studies have been carried out to reduce the mutual coupling between the multiple antenna elements. A corrugated ground plane with $\lambda / 4$ slot was used to reduce the interference of a current flowing in the common ground plane [9]. In [10], the protruded T-shaped stub and Lshaped stub at ground plane are used to reduce the mutual coupling between two element antennas. The polarization diversity technique [11] and defected ground structure [12] are adopted to improve the separation between the element antennas. Various MIMO antennas for laptop or mobile handset applications were provided during these years. The majority of them are designed for laptop operating at WLAN $(2.4-2.48 \mathrm{GHz}, 5.2 \mathrm{GHz})$ and WiMAX band [1315]. MIMO antennas for mobile terminal have drawn more and more research interest nowadays, and more and more designs are presented. Most of MIMO antennas for handset applications are designed to resonate at high frequencies such as GSM1800/1900/UMTS [9-12]. However, fewer researches were carried out to design MIMO antennas resonating at lower frequencies such as GSM850/900/LTE 700 or to design MIMO antennas resonating at both lower frequencies and higher frequencies. In $[16,17]$, two articles of MIMO antennas for only LTE 700 application were provided.

In this paper, we present a promising small-size on-board printed multiple-input and multiple-output 

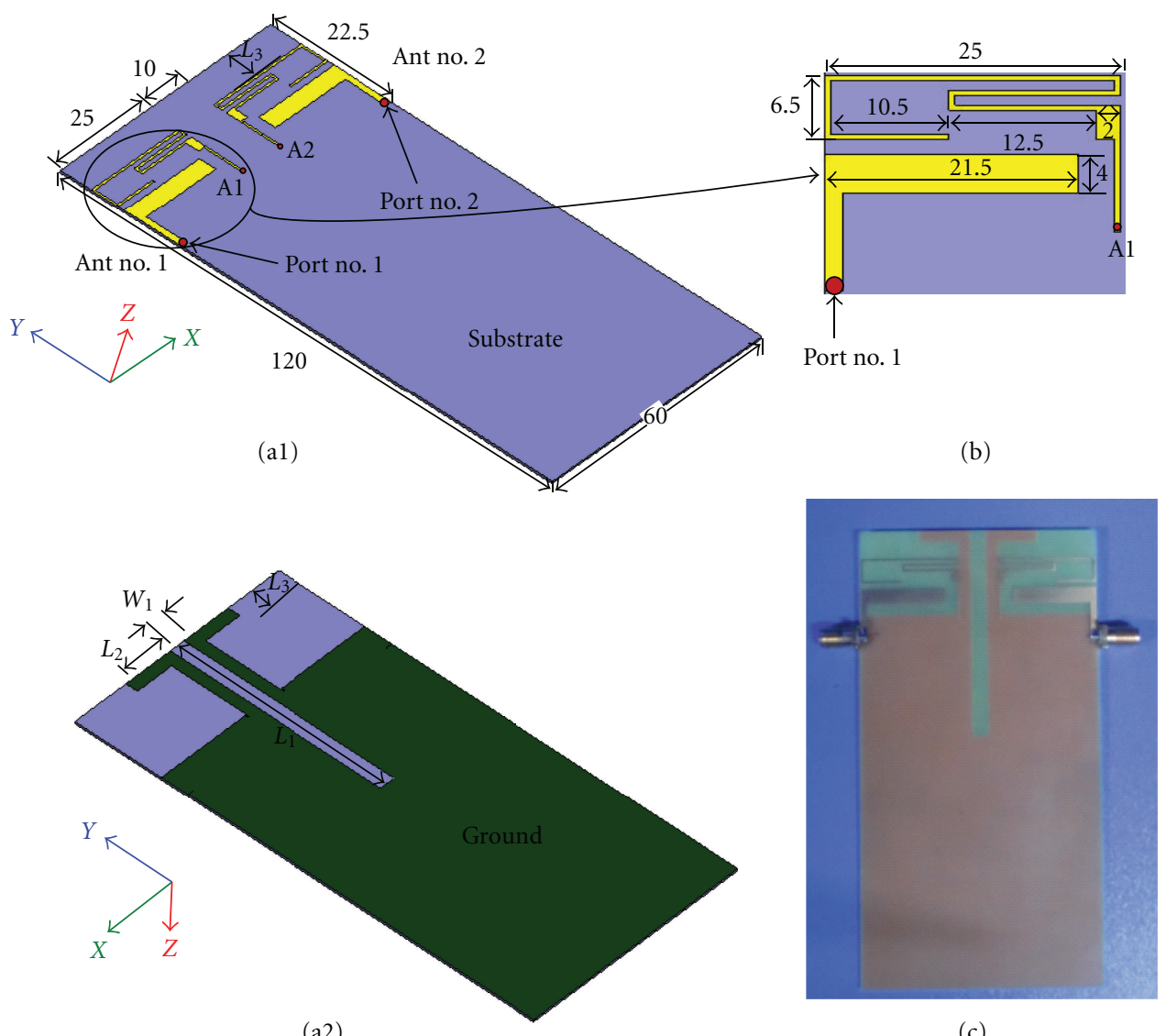

(c)

FIGURE 1: Geometry of the proposed MIMO antenna: (a1) and (a2) 3D view of the proposed MIMO antenna, (b) element antenna structure, and (c) fabricated MIMO antenna.

antenna for tetraband wireless communication applications (GSM900/1800/1900/UMTS). The proposed antenna, which consists of two coupled-fed loop antennas with symmetric configuration, was printed on a $120 * 60 * 0.8 \mathrm{~mm}^{3}$ FR-4 substrate of relative permittivity of 4.4 , loss tangent 0.02 . Two coupled-fed strip antennas were etched on the top layer of the substrate. The grounds with a $4 \mathrm{~mm}$-width slot and dual-inverted-L-shaped ground branches were etched on the bottom layer of the substrate. The loop antenna is formed by a loop strip with end terminal short-circuited to the ground plane and its front section capacitively coupled to a feeding strip which is also an efficient radiator to contribute a resonant mode for the antenna's upper band to cover 1710$2170 \mathrm{MHz}$. Through the coupling excitation, the antenna can also generate a qua-wavelength loop resonant mode to form the antenna's lower band to cover the $880-960 \mathrm{MHz}$ [18]. The isolation between the two antenna elements highly improved when a slot and a dual-inverted-L were added on the ground. The slot on the ground also generates a resonance at about $900 \mathrm{MHz}$ that broaden the antenna's lower operating band.

\section{Antenna Design}

The geometry of the proposed MIMO antenna is shown in Figure 1. Two element antennas and ground were etched on the top layer and bottom layer, respectively. Figure 1(a1) depicts the layout of the two coupled-fed loop antennas; the edge-to-edge spacing is $10 \mathrm{~mm}$. The detailed dimension of the element antenna is shown in Figure 1(b). Each element antenna occupies a footprint of $22.5 * 25 \mathrm{~mm}^{2}$. The element antenna is formed by a $0.5 \mathrm{~mm}$-width loop strip and a feeding strip. The end terminal of the loop strip is short-circuited to the ground via pin A1 and A2. A $2 * 4 \mathrm{~mm}^{2}$ rectangular matching piece was added to improve the impedance matching performance. The front section of the loop strip is capacitively coupled to the feeding strip which starts from a shot section of $50 \Omega$ microstrip. The feeding strip also generates a resonant mode for GSM1800/1900/UMTS.

The ground of the proposed antenna is shown in Figure 1(a2). The substrate extends its length by $L_{3}=$ $7.5 \mathrm{~mm}$. A slot with length $L_{1}=55 \mathrm{~mm}$ and width $W_{1}=$ $4 \mathrm{~mm}$ was laid in the middle of the ground plane. A dualinverted-L-shaped ground branch with strip width $W_{2}=$ $3 \mathrm{~mm}$ and $x$-direction end section length $L_{2}=15 \mathrm{~mm}$ was laid on the left and right sides of the slot.

Figure $1(\mathrm{c})$ is the photograph of the fabricated MIMO antenna. Port no. 1 and port no. 2 were connected with two SMA female connectors for $S$-parameter measurements and radiation patterns measurements. 


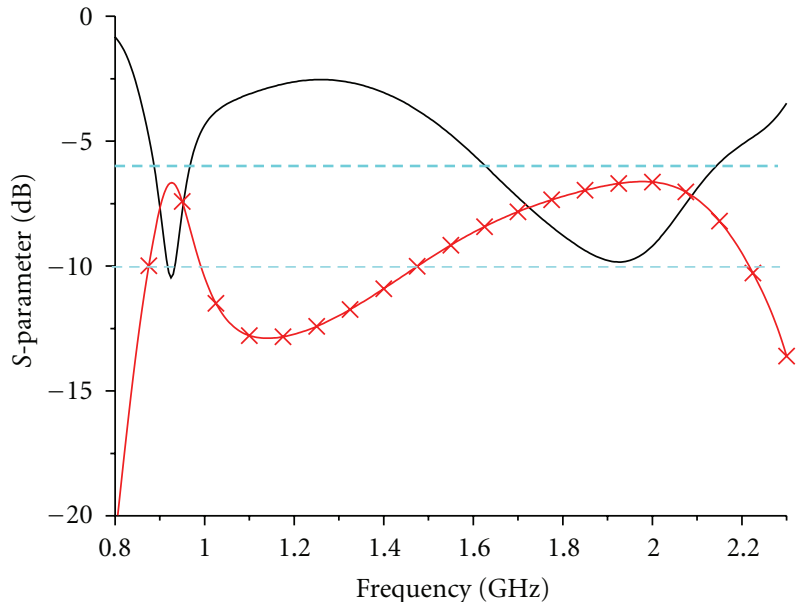

- $S_{11}$-Without_Decoupling $\star S_{21-W i t h o u t \_D e c o u p l i n g}$

(a)

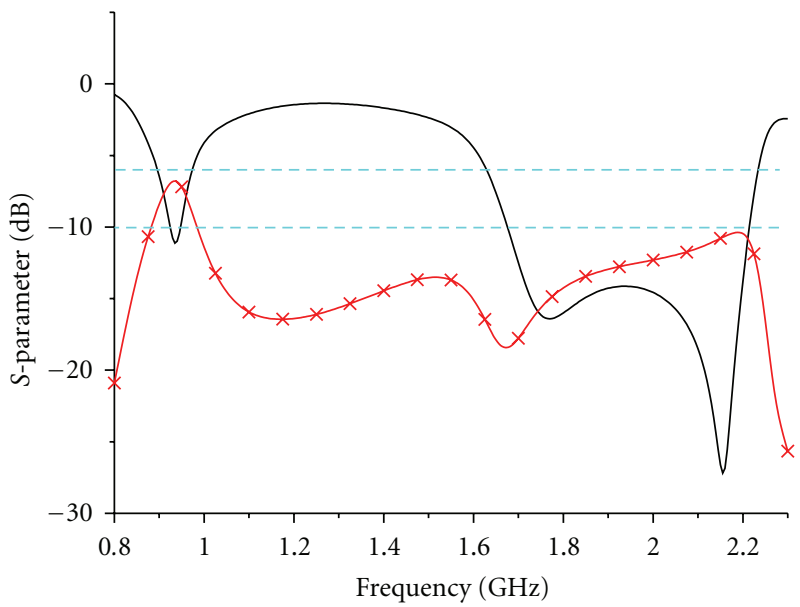

- $S_{11}$ Dual-inverted-L

$\leftarrow S_{21}$ DDual-inverted-L

(c)

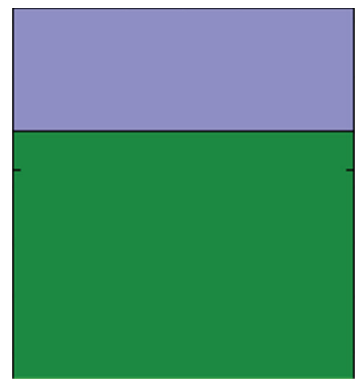

(a)
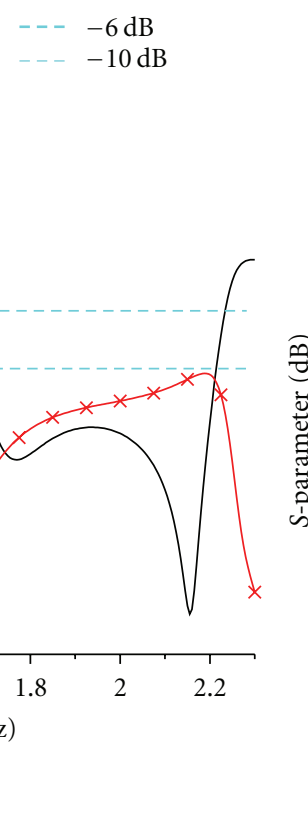

(c)
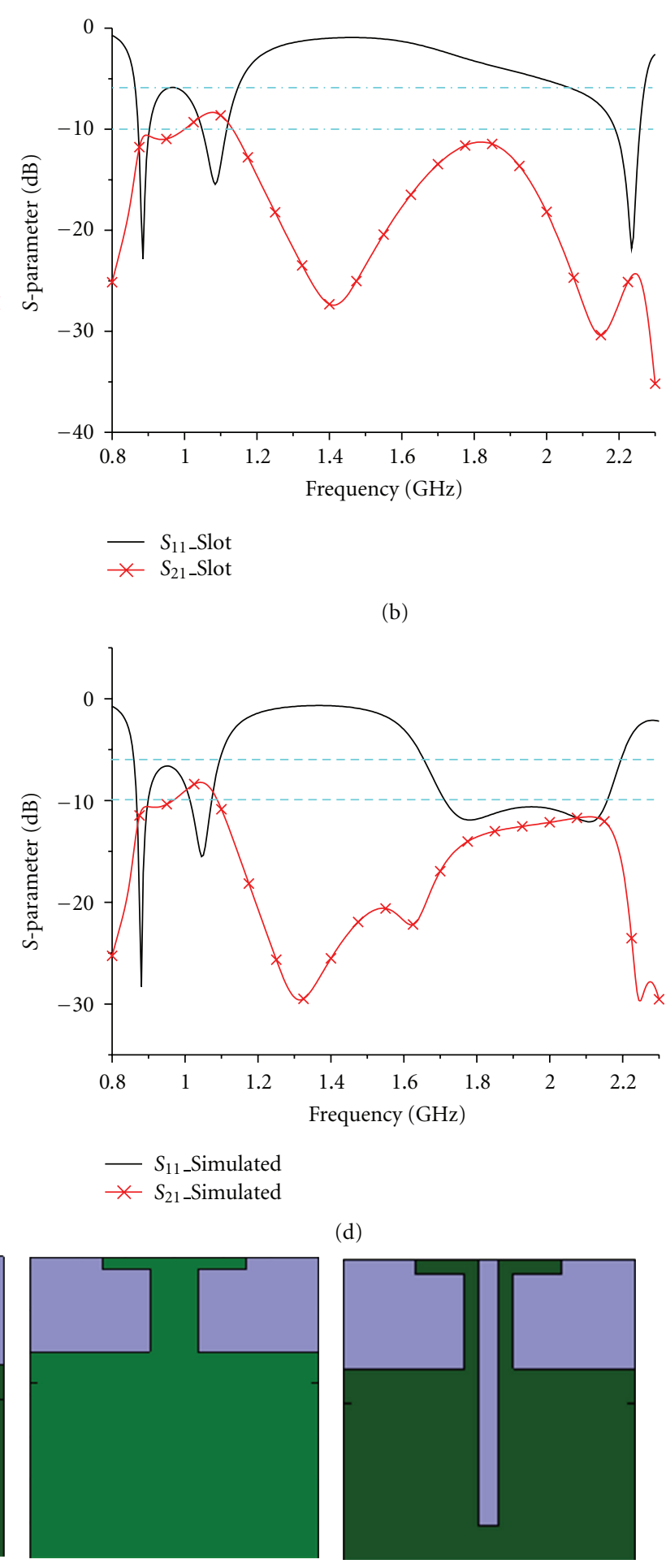

(b)

(d)

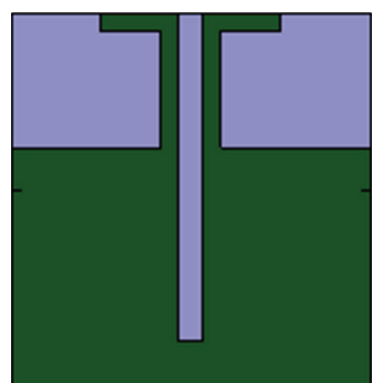

(d)

FIGURE 2: Simulated $S_{11}$ and $S_{21}$ curves: (a) simulated $S$-parameter curves of the antenna without slot on ground plane and ground branch, (b) simulated $S$-parameter curves of antenna with slot on the ground plane, (c) simulated $S$-parameter of antenna with dual-inverted-L shaped ground branch, (d) simulated $S$-parameter of antenna with both slot and ground branch. 

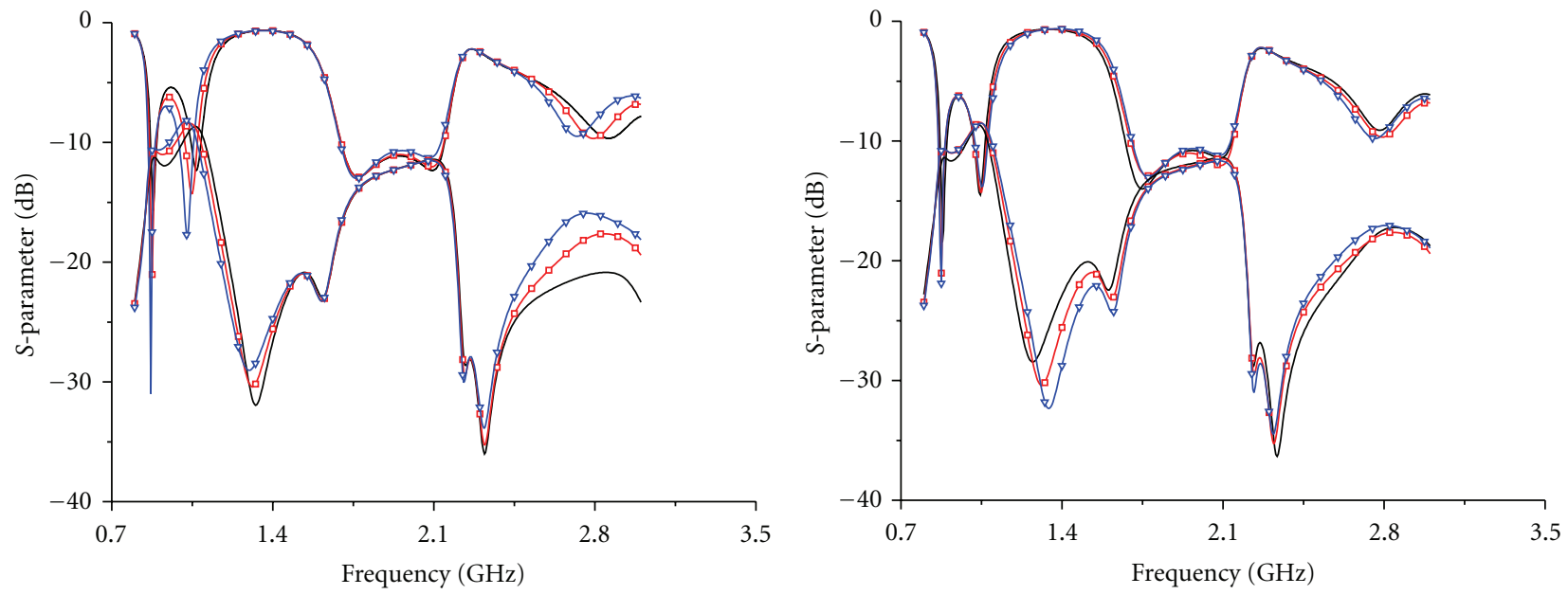

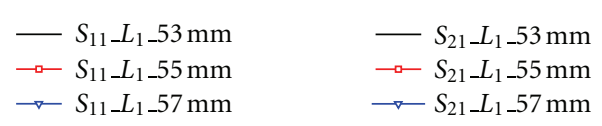

(a)

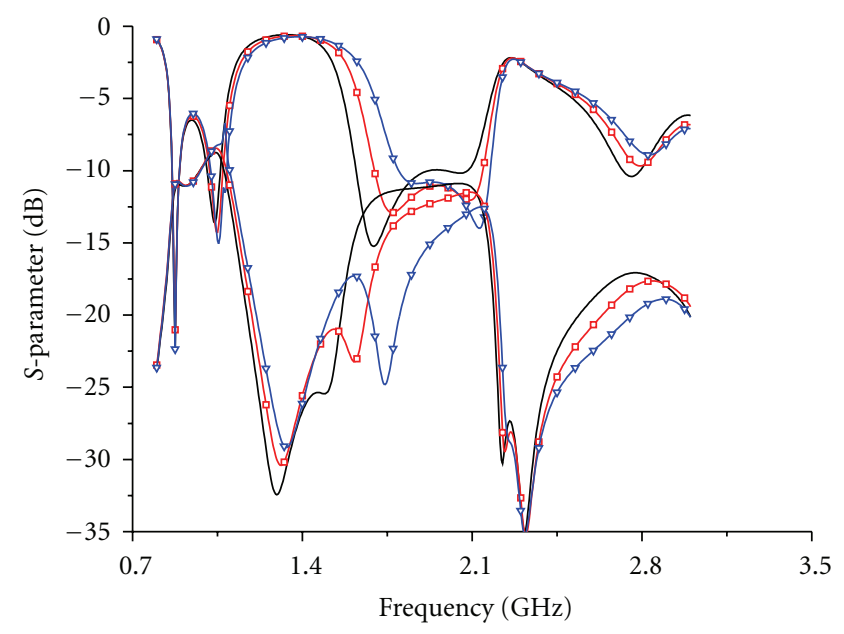

(c)

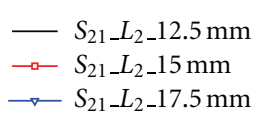

)

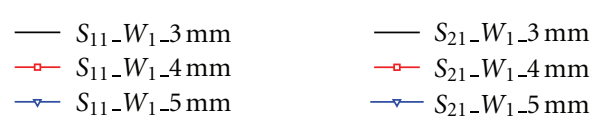

(b)
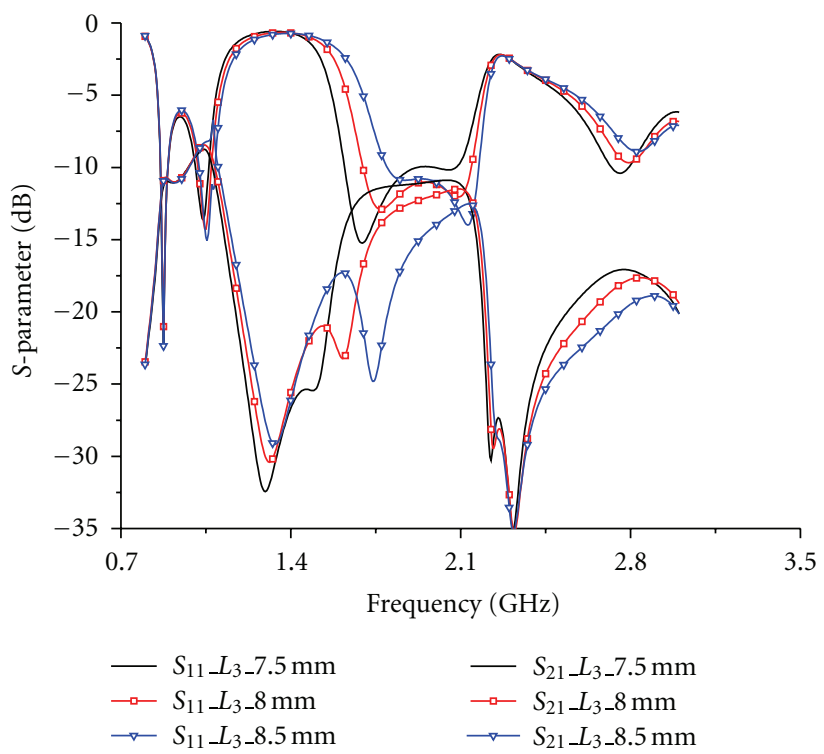

(d)

Figure 3: Parameter studies of $L 1 / W 1 / L 2$ and $L 3$; the nominal values of the dimensions are $L_{1}=55 \mathrm{~mm} / W_{1}=4 \mathrm{~mm} / L_{2}=15 \mathrm{~mm} / L_{3}=$ $8 \mathrm{~mm}$.

\section{Simulation Analysis}

The antenna was simulated and optimized with 3D fullwave EM simulation software Ansoft HFSS (High Frequency Structure Simulator) V11 [19]. Various simulations were carried out to verify different decoupling techniques, and simulation results were obtained. $S_{11} \& S_{22}$ or $S_{21} \& S_{12}$ are the same because of the absolutely symmetrical structure. Figure 2 shows the simulated $S_{11}$ and $S_{21}$ curves of antenna with different ground planes. Figure 2(a) is the simulated $S_{11}$ and $S_{21}$ curves of antenna without decoupling structure. Both the lower and upper frequency bands can merely satisfy the impedance matching bandwidth requirement that
$S_{11}<-6 \mathrm{~dB}$ frequency band should cover $880-960 \mathrm{MHz}$ and $1710-2170 \mathrm{MHz}$. However, the $S_{21}$ values are larger than $-7 \mathrm{~dB}$ at both GSM900 and GSM1800/1900/UMTS bands. Figure 2(b) shows the $S$-parameters of the antenna with a slot in the middle of the ground plane to decrease the mutual coupling between the two element antennas. The isolation at both lower and higher frequency bands improved to $10 \mathrm{~dB}$, but the upper resonant mode shifted to $2.2 \mathrm{GHz}$ with only about $150 \mathrm{MHz} S_{11}<-6 \mathrm{~dB}$ bandwidth. Additionally, the inserted slot on the ground plane contributes to a new resonance at $1.05 \mathrm{GHz}$ which could be shifted to lower frequency and broaden the lower frequency operating bandwidth by adjusting the length of the slot $\left(L_{2}\right)$. Impedance matching 

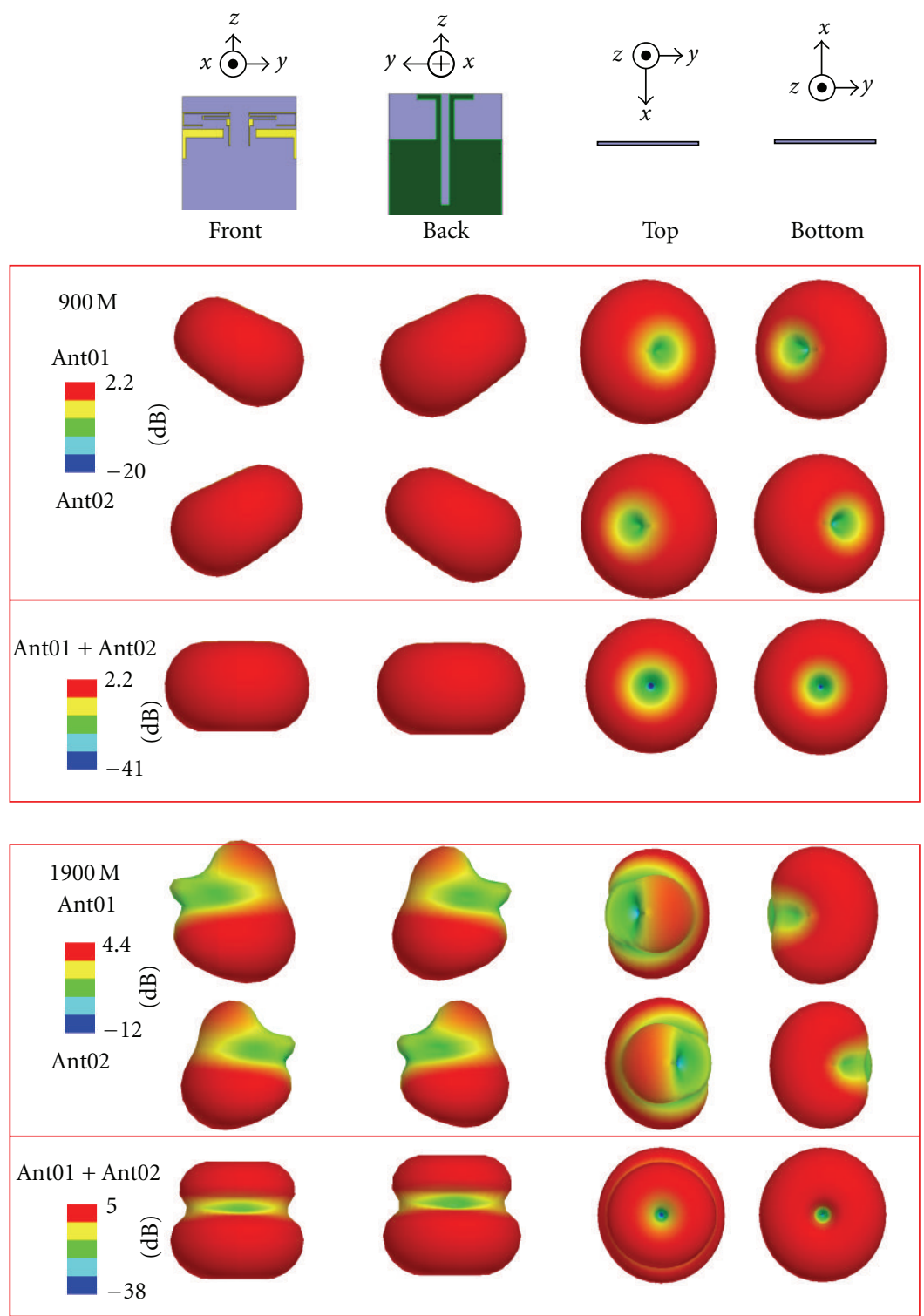

FIgURE 4: Simulated total power 3D radiation pattern of antenna no. 1 and antenna no. 2 at $900 \mathrm{MHz}$ and $1900 \mathrm{MHz}$.

performance of antenna with dual-inverted-L ground branch is depicted in Figure 2(c). Both the Return Loss and Isolation satisfy the requirements at upper frequency band when dual-inverted-L ground branch was added in the ground. But the dual-inverted-L ground branch structure does not improve Isolation at the lower frequency band. The Isolation maintains $7 \mathrm{~dB}$ at about $900 \mathrm{MHz}$. The Isolation and Return Loss satisfied the requirements at both the upper and lower frequency when two decoupling techniques combined. Figure 2(d) shows the simulated $S_{11}$ and $S_{21}$ curves of the antenna with both slot and dual-inverted-L-shaped ground branch. The $S_{11}<-6 \mathrm{~dB}$ frequency bandwidth covers from $865 \mathrm{MHz}$ to $1100 \mathrm{MHz}$ at lower frequency band and from $1650 \mathrm{MHz}$ to $2190 \mathrm{MHz}$ at upper frequency band. The $S_{21}<$ $-10 \mathrm{~dB}$ frequency band spans from $800 \mathrm{MHz}$ to $965 \mathrm{MHz}$ at lower frequency band and the entire upper frequency band.
All the dimensions that may affect the return Loss and isolation performances of the antenna are examined. Figure 1(a2) shows the dimensions that influence the antenna's return loss and isolation performances. $L_{1}$ is the length of the slot, and $W_{1}$ is the width of the slot. $L_{2}$ is the length of the inverted-L stub's $x$-direction end section. $L_{3}$ represents the extended length of the PCB. Figure 3 shows how all the four dimensions above affect the $S$-parameters of the antenna while other dimensions did not vary. The nominal values of the four dimensions are $L_{1}=55 \mathrm{~mm} / L_{2}=$ $15 \mathrm{~mm} / L_{3}=7.5 \mathrm{~mm} / W_{1}=4 \mathrm{~mm}$. It is difficult to choose a set of dimensions to meet the antenna's requirements because $S_{11}$ and $S_{21}$ curves interact with each other at the lower frequency band. A set of dimensions were obtained after studying all the dimensions, $L_{1}=55 \mathrm{~mm} / L_{2}=$ $15 \mathrm{~mm} / L_{3}=7.5 \mathrm{~mm} / W_{1}=4 \mathrm{~mm}$. 


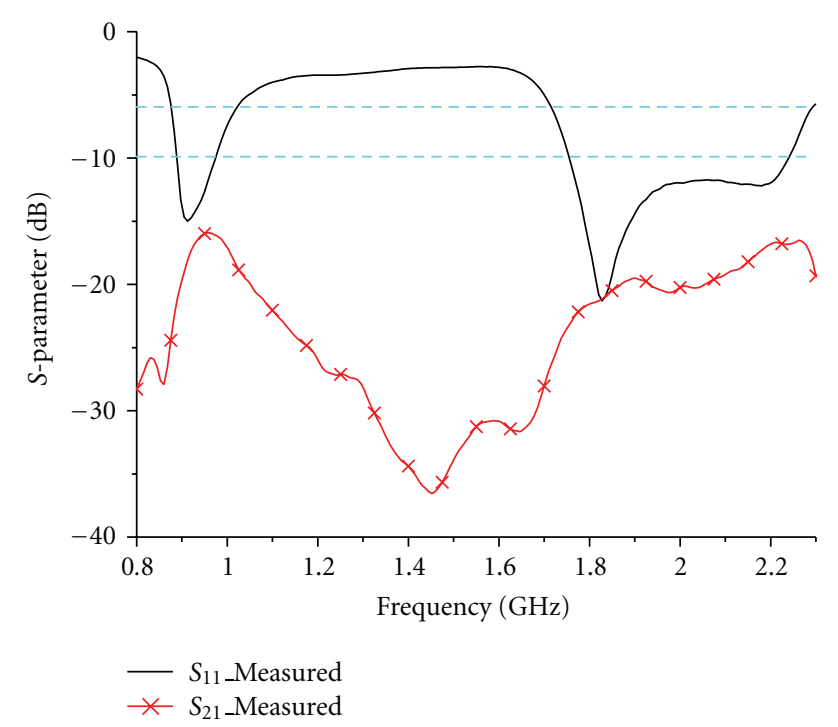

Figure 5: Measured S11 and S21 Curves.

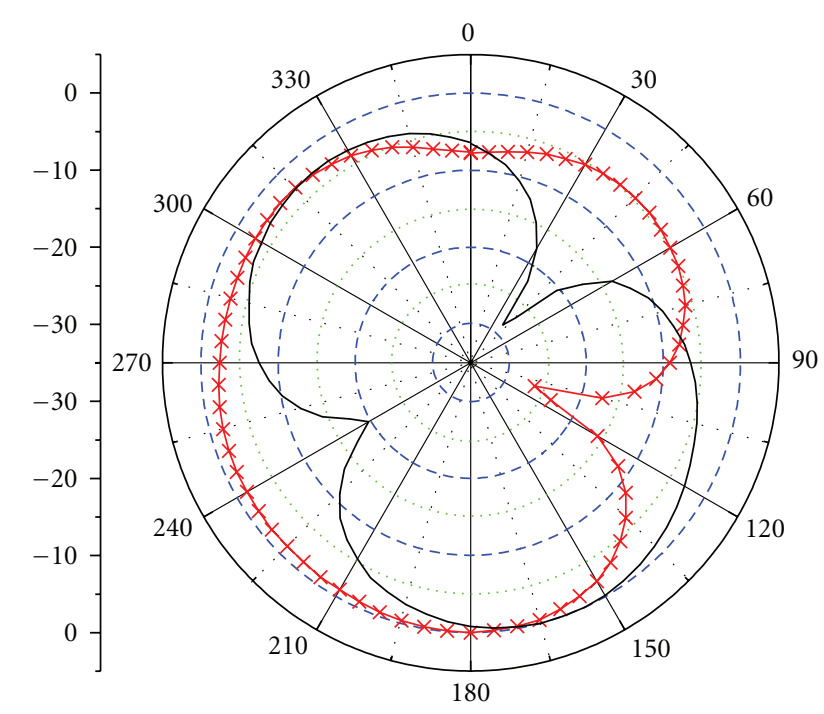

- Ant01_E-plane_Horizontal

$\leftarrow$ Ant01_E-plane_Vertical

Figure 6: Measured E-Plane radiation pattern of antenna no. 1 at $900 \mathrm{MHz}$.

The simulated three-dimensional (3D) total power radiation patterns at $900 \mathrm{MHz}$ and $1900 \mathrm{MHz}$ of the proposed small-size MIMO antenna are plotted in Figure 4. The radiation patterns are seen from four different directions (front, back, top, and bottom) considering the cases of element antennas work respectively and two element antennas work together. For the lower frequency at $900 \mathrm{MHz}$, each element antenna generates an oblique dipole-like radiation pattern with the center axes orthogonal to each other. Two bolique dipole-like radiation patterns combined to a unique dipolelike radiation patterns with it center axis directly oriented toward $z$-axis. The same diversity performance could also be

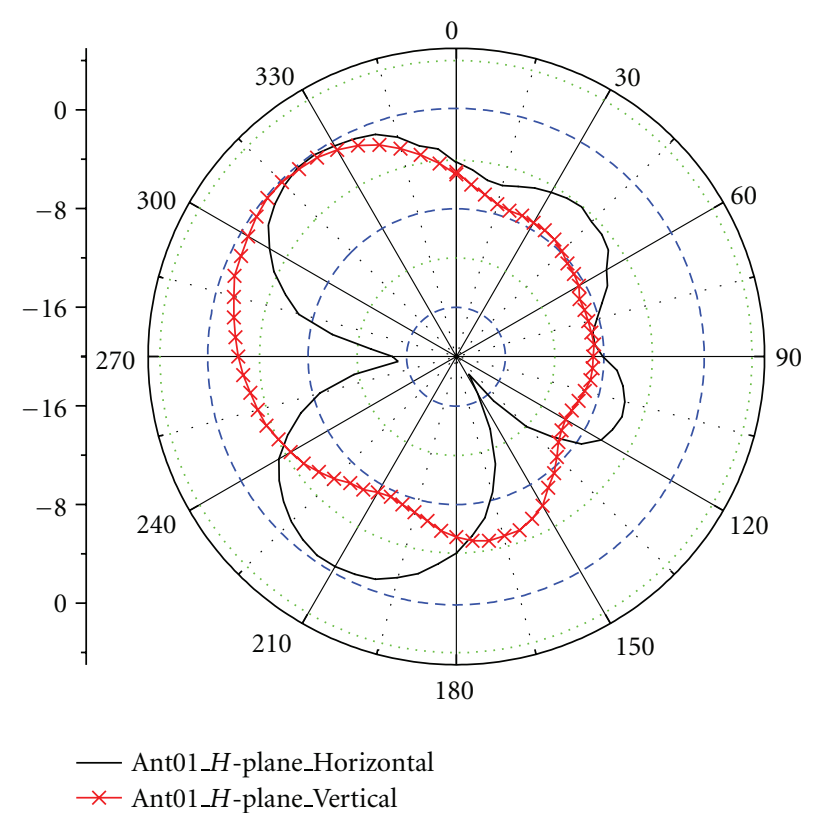

Figure 7: Measured $H$-Plane radiation pattern of antenna no. 1 at $900 \mathrm{MHz}$.

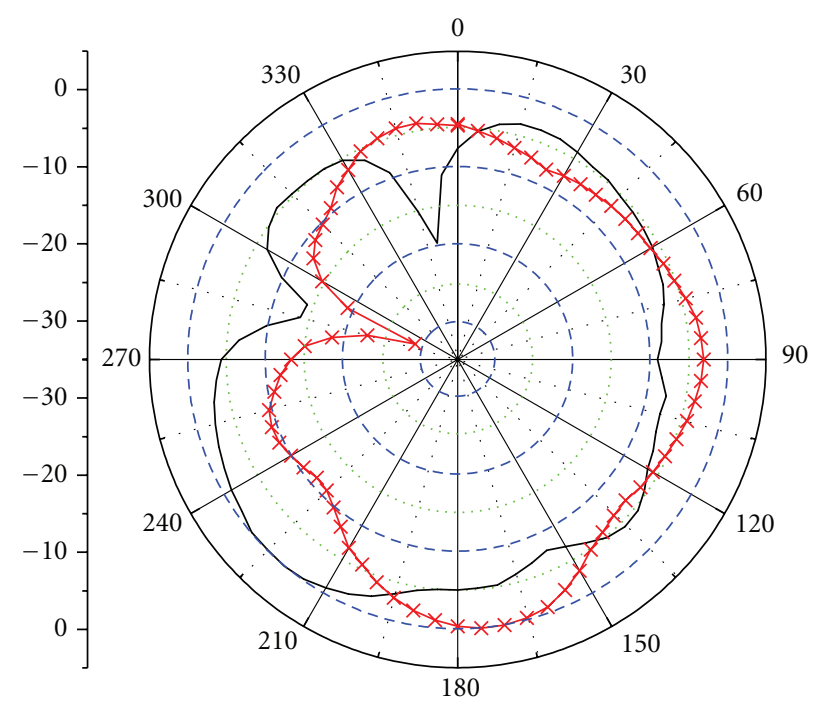

- Ant01_E-plane_Horizontal
$*$ Ant01_E-plane_Vertical

Figure 8: Measured E-Plane radiation pattern of antenna no. 1 at $1900 \mathrm{MHz}$.

seen from the radiation pattern at $1900 \mathrm{MHz}$. Two radiation patterns with dips and nulls combined to a dual-circle dipole-like radiation pattern with the axis toward $z$-axis. The diversity characteristic enables the proposed MIMO antenna a promising design for mobile handset applications. The simulated peak gains of the element antennas are about $2.2 \mathrm{dBi}$ at $900 \mathrm{MHz}$ and $4.4 \mathrm{dBi}$ at $1900 \mathrm{MHz}$. 


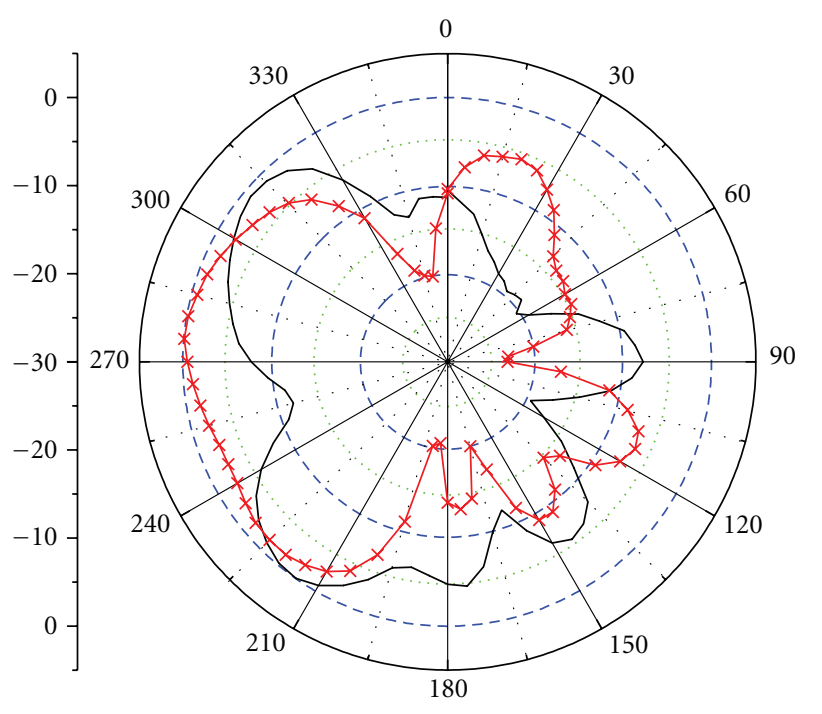

- Ant01_H-plane_Horizontal

$*$ Ant01_H-plane_Vertical

Figure 9: Measured $H$-Plane radiation pattern of antenna no. 1 at $1900 \mathrm{MHz}$.

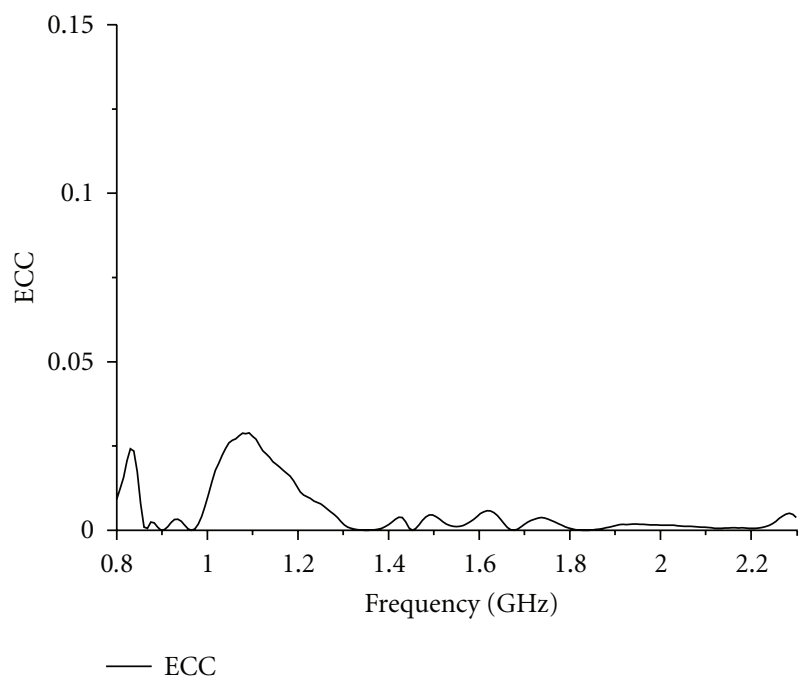

Figure 10: Calculated ECC Curve.

\section{Measurement Result}

The antenna was fabricated and tested in the school of Electronic Engineering of Beijing University of Posts and Telecommunications (BUPT). The $S$-parameters were measured by a Vector Network Analyzer, and the radiation pattern measurement is carried out inside an anechoic chamber.

The measured $S_{11}$ and $S_{21}$ curves of the antenna are plotted in Figure 5. The $S_{11}<-6 \mathrm{~dB}$ frequency bandwidth covers from $870 \mathrm{MHz}$ to $1010 \mathrm{MHz}$ at lower frequency band and from $1710 \mathrm{MHz}$ to $2290 \mathrm{MHz}$ at higher frequency band. The measured $S_{21}$ values of the MIMO antenna are smaller than $-15 \mathrm{~dB}$ over the entire frequency band of $800 \mathrm{MHz}-$ $2300 \mathrm{MHz}$. The return loss and isolation of the antenna fully satisfies the requirement of MIMO antenna operation at GSM900/1800/1900/UMTS.

The $900 \mathrm{MHz}$ and $1900 \mathrm{MHz}$ measured radiation patterns of antenna no. 1 are depicted in Figures 6, 7, 8, and 9. The measured $E$-Plane and $H$-Plane radiation patterns of antenna no. 2 are consistent with radiation patterns of antenna no. 1 except a 180-degree rotation because of the exactly symmetrical configuration. Each figure shows the radiation pattern at one frequency including $H$-plane's ( $x$ $y$ plane)/E-plane's ( $x-z$ plane) horizontal radiation pattern and vertical radiation pattern.

The ECC (envelope correlation coefficient) is usually used to evaluate the diversity capability of a multiantenna system and should ideally be computed using the 3D radiation pattern [20]. Assuming that the antennas will operate in a uniform multipath environment, it can be alternatively calculated by using the scattering parameters. The ECC of two antennas is given by (1) [21]. The calculated envelope correlation coefficient (ECC) curve is plotted in Figure 10 to evaluate the performance of the MIMO antenna. The ECCs of the two element antennas are always below 0.05 over the whole frequency band. This leads to perfect performance in terms of diversity:

$$
\rho_{12}=\frac{\left|S_{11}^{*} S_{12}+S_{12}^{*} S_{22}\right|}{\left(1-\left|S_{11}\right|^{2}-\left|S_{21}\right|^{2}\right)\left(1-\left|S_{22}\right|^{2}-\left|S_{12}\right|^{2}\right)} .
$$

\section{Conclusion}

A dual-element small-size printed strip multiple-input and multiple-output (MIMO) antenna was proposed in this paper. The edge-to-edge spacing between the two elements is only $0.03 \lambda_{0}$ of $920 \mathrm{MHz}$. A ground plane with a slot and dual-inverted-L-shaped stub were used to decrease the mutual coupling between the element antennas. Antenna performances of with- and without-decoupling techniques were listed. Prototypes were fabricated and measured after parameter optimizations. The measured $-6 \mathrm{~dB}$ bandwidth are $870 \mathrm{MHz}$ to $1010 \mathrm{MHz}$ and $1710 \mathrm{MHz}$ to $2290 \mathrm{MHz}$. The isolations are better than $15 \mathrm{~dB}$ covering all the frequency band. The simulated and measured radiation patterns of the antenna show good diversity characteristic. The calculated ECCs are below 0.05 over the whole band. The features above proved that the proposed antenna is a promising product for mobile terminals.

The $S_{11}<-6 \mathrm{~dB}$ frequency bandwidth covers from $870 \mathrm{MHz}$ to $1010 \mathrm{MHz}$ at lower frequency band and from $1710 \mathrm{MHz}$ to $2290 \mathrm{MHz}$ at higher frequency band.

\section{Acknowledgment}

This work was supported in part by the Fundamental Research Funds for the Central Universities, the National Natural Science Foundation of China under Grant nos. 
60703036 and 41040040, and China Important National Science and Technology Specific Project with no. 2009ZX03007003-01.

\section{References}

[1] Wikipedia, http://en.wikipedia.org/wiki/LTE.

[2] Wikipedia, http://en.wikipedia.org/wiki/4G.

[3] Wikipedia, http://en.wikipedia.org/wiki/MIMO.

[4] J. W. Wallace, M. A. Jensen, A. L. Swindlehurst, and B. D. Jeffs, "Experimental characterization of the MIMO wireless channel: data acquisition and analysis," IEEE Transactions on Wireless Communications, vol. 2, no. 2, pp. 335-343, 2003.

[5] S. C. K. Ko and R. D. Murch, "Compact integrated diversity antenna for wireless communications," IEEE Transactions on Antennas and Propagation, vol. 49, no. 6, pp. 954-960, 2001.

[6] G. J. Foschini and M. J. Gans, "On limits of wireless communications in a fading environment when using multiple antennas," Wireless Personal Communications, vol. 6, no. 3, pp. 311335, 1998.

[7] Y. Gao, X. Chen, Z. Ying, and C. Parini, "Design and performance investigation of a dual-element PIFA array at $2.5 \mathrm{GHz}$ for MIMO terminal," IEEE Transactions on Antennas and Propagation, vol. 55, no. 12, pp. 3433-3441, 2007.

[8] A. Derneryd and G. Kristensson, "Signal correlation including antenna coupling," Electronics Letters, vol. 40, no. 3, pp. 157$159,2004$.

[9] M. Karakoikis, C. Soras, G. Tsachtsiris, and V. Makios, "Compact dual-printed inverted-F antenna diversity systems for portable wireless devices," IEEE Antennas and Wireless Propagation Letters, vol. 3, no. 1, pp. 9-14, 2004.

[10] Y. Ding, Z. Du, K. Gong, and Z. Feng, "A novel dualband printed diversity antenna for mobile terminals," IEEE Transactions on Antennas and Propagation, vol. 55, no. 7, pp. 2088-2096, 2007.

[11] S. B. Yeap, X. Chen, J. A. Dupuy, C. C. Chiau, and C. G. Parini, "Low profile diversity antenna for MIMO applications," Electronics Letters, vol. 42, no. 2, pp. 69-71, 2006.

[12] M. Salehi, A. Motevasselian, A. Tavakoli, and T. Heidari, "Mutual coupling reduction of microstrip antennas using defected ground structure," in Proceedings of the 10th IEEE Singapore International Conference on Communications Systems (ICCS '06), pp. 1-5, November 2006.

[13] C. Y. Chiu, C. H. Cheng, R. D. Murch, and C. R. Rowell, "Reduction of mutual coupling between closely-packed antenna elements," IEEE Transactions on Antennas and Propagation, vol. 55, no. 6, pp. 1732-1738, 2007.

[14] A. C. K. Mak, C. R. Rowell, and R. D. Murch, "Isolation enhancement between two closely packed antennas," IEEE Transactions on Antennas and Propagation, vol. 56, no. 11, pp. 3411-3419, 2008.

[15] S. Zhang, S. N. Khan, and S. He, "Reducing mutual coupling for an extremely closely-packed tunable dual-element PIFA array through a resonant slot antenna formed in-between," IEEE Transactions on Antennas and Propagation, vol. 58, no. 8, Article ID 5466048, pp. 2771-2776, 2010.

[16] H. Bae, F. J. Harackiewicz, M. J. Park et al., "Compact mobile handset MIMO antenna for LTE700 applications," Microwave and Optical Technology Letters, vol. 52, no. 11, pp. 2419-2422, 2010.

[17] M. Han and J. Choi, "Small-size printed strip MIMO antenna for next generation mobile handset application," Microwave and Optical Technology Letters, vol. 53, no. 2, pp. 348-352, 2011.

[18] K. L. Wong, W. Y. Chen, and T. W. Kang, "On-board printed coupled-fed loop antenna in close proximity to the surrounding ground plane for penta-band WWAN mobile phone," IEEE Transactions on Antennas and Propagation, vol. 59, no. 3, pp. 751-757, 2011.

[19] Ansoft Corporation HFSS [online], http://www.ansoft.com/ products/hf/hfss.

[20] I. Salonen and P. Vainikainen, "Estimation of signal correlation in antenna arrays," in Proceedings of the 12th International Symposium Antennas, vol. 2, pp. 383-386, July 2002.

[21] S. Blanch, J. Romeu, and I. Corbella, "Exact representation of antenna system diversity performance from input parameter description," Electronics Letters, vol. 39, no. 9, pp. 705-707, 2003. 

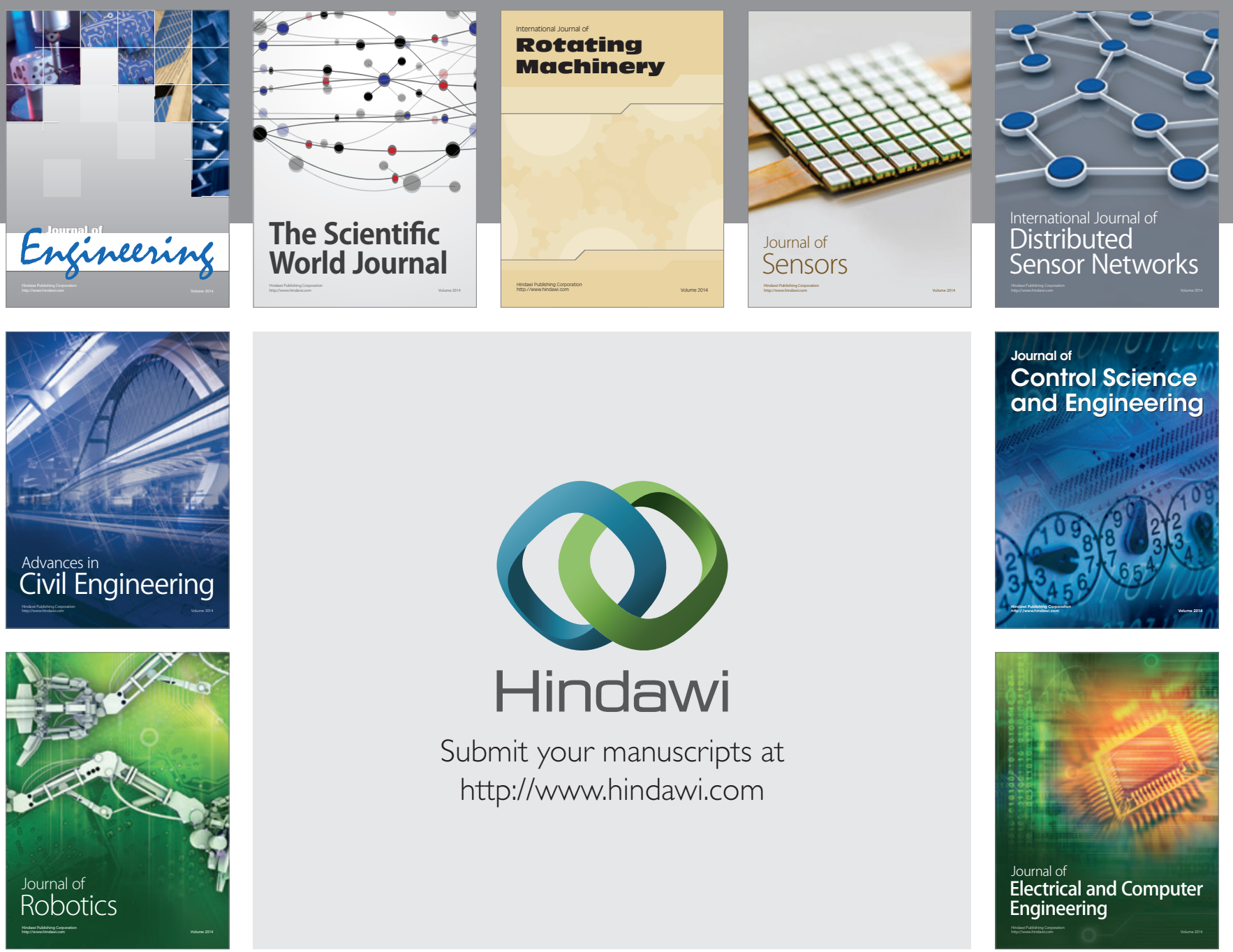

Submit your manuscripts at

http://www.hindawi.com
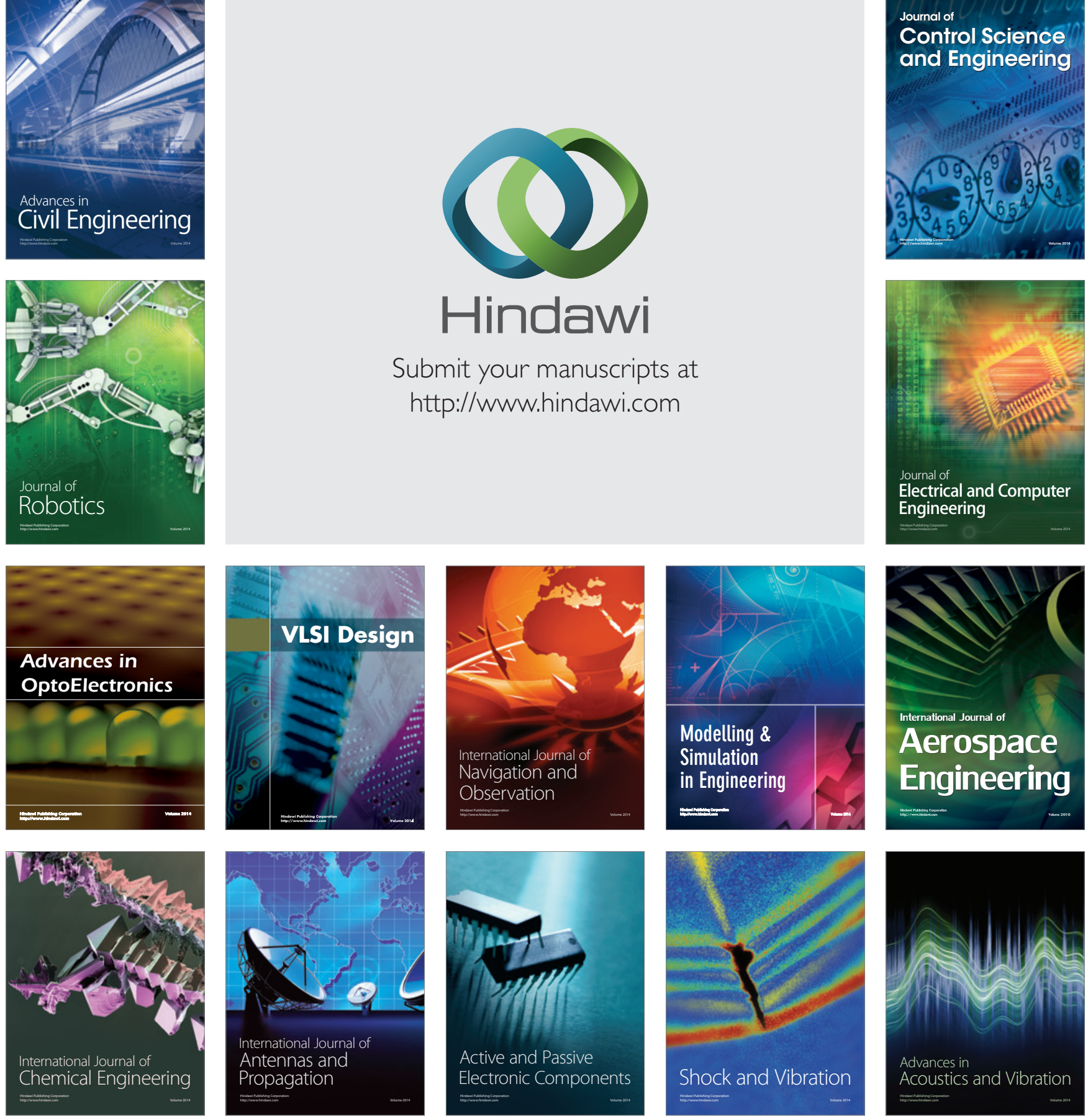